\title{
Albrecht Göschel \\ Wandlungen kultureller Orientierungen \\ in der Abfolge von Generationen
}

Zusammenfassung: Der Bereich der Kultur im engen Sinne, also die ästhetischsymbolische Produktion, kann in einer Phase der Auflösung traditioneller Schichten als Instrument der Distanzierung auch zwischen anderen Gruppierungen, also z.B. zwischen Generationen angesehen werden. Beim Vergleich von vier bundesdeutschen Generationen werden deutliche Unterschiede in den kulturellen Orientierungen erkennbar, die als »Generationshabitus « verstanden werden können. Trotz der Gegensätze zwischen den aufeinanderfolgenden Generationen stehen diese aber auch in einer kontinuierlichen Entwicklung, die sich als Verwestlichung des Kulturverständnisses beschreiben läßt. Damit wird deutlich, daß der deutsche »kulturelle Sonderweg «, in dem Kultur als säkulare Religion galt, in den 60er/70er Jahren zugunsten einer Dienstleistungshaltung gegenüber Kulturleistungen beendet wird, wie sie auch für andere westliche und vor allem für die angelsächsischen Länder kennzeichnend ist.

\section{Untersuchungsannahmen und Methoden}

Der Bereich der Kultur im engeren Sinne, also der ästhetischen-symbolischen Produkte und ihrer Rezeption, kann, wie z.B. von Bourdieu (1982) gezeigt, als Sphäre der Distanzierung zwischen konkurrierenden Gruppen angesehen werden. Als solche Gruppen gelten i.d.R. Schichten oder Lebensstile. Daß auch Generationen in ihrer Abfolge in Kokurrenzbeziehungen stehen, ist von Elias (1989:319) mit Nachdruck betont worden. Dabei zielt er nicht auf Generationskonflikte im landläufigen Sinne, also auf innerfamiliäre Ablösungsprozesse der Kinder von ihren Eltern, sondern auf Machtkonkurrenzen zwischen älteren und jüngeren Mitgliedern einer Gesellschaft. Nachrückende Generationen oder Kohorten finden jeweils die entscheidenden Positionen eines Machtgefälles besetzt, und sind damit daran gehindert, innovativ zu werden. Es können sogar die Einstiegsstellen in Karrieren versperrt sein, wenn durch demographische Schwankungen einzelne Generationen übermäßig stark und auf lange Zeit in den Aufstiegslinien vertreten sind. Derartige Situationen hat es in der jüngeren deutschen Entwicklung mehrfach gegeben, z.B. ab Mitte der 70er Jahre für viele Hochschulabsolventen. Es liegt nahe, für solche Situationen Generationskonflikte anzunehmen, die sich, gerade weil sie sich als Konflikte um Besitzstände und Positionen und nicht als Auseinandersetzungen um unterschiedliche Organisationsformen der Macht- und Besitzverteilung entwickeln, in ausgeprägten symbolischen 
Konkurrenzen, also als Konflikte im Bereich der Kultur ausdrücken, verbunden allerdings mit Lebensformkonflikten, also einem Gesamtkomplex von Symbolen. Von diesen Annahmen ging das Forschungsvorhaben »Wandlungen kultureller Orientierungen « aus, das zur Zeit am Deutschen Institut für Urbanistik durchgeführt wird. Das Projekt ist noch nicht abgeschlossen, so daß hier nur erste Eindrücke wiedergegeben werden können. Das Forschungsinteresse zielt dabei auf die Einstellung zu Kultur im engeren Sinne, also zu künstlerischen Leistungen und Produkten und zu Kultureinrichtungen wie Theatern, Kinos, Museen, Konzerthäusern usw.

Die empirische Grundlage der folgenden Aussagen bilden ca. 60 Leitfadeninterviews mit Besuchern von Einrichtungen, ca. 40 Expertengespräche und als Kern der Untersuchung bisher 24 offene, nicht-direktive, biographische Interviews. Während bei den Leitfadeninterviews eine breitere Streuung der Schulabschlüsse angestrebt wurde, sind bei den offenen biographischen Interviews nur Personen mit Hochschulabschlüssen befragt worden. Diese Einschränkung mindert ohne Zweifel den Repräsentativitätsanspruch der Studie, war jedoch aus Kapazitätsgründen notwendig. Aber auch inhaltlich ist sie zu begründen. Nach wie vor werden Kultureinrichtungen und Kunstgüter in der Bundesrepublik nur von, einer Minderheit der Bevölkerung mit hohen Bildungsabschlüssen genutzt bzw. zur Kenntnis genommen. Es erschien daher gerechtfertigt, sich in dieser Untersuchung, die sich als Pilotstudie versteht, vorerst auf diese Gruppen zu konzentrieren. Gegen die Annahme eines kulturellen Wandels aus Konkurrenzen zwischen Generationen, also aus Gegensätzen, könnte die übliche Vorstellung sprechen, daß gerade Orientierungen in diesem Bereich Traditionen unterliegen. Nach dieser Vorstellung würden ähnliche Präferenzen und Einstellungen innerhalb der Schichten eher konstant weitergegeben werden, auch wenn diese Kontinuität und Stabilität begleitet wird von einem permanenten Vorgang der innerfamiliären Generationskonflikte, die als ständig notwendige Emanzipation der Jüngeren und nicht als Konflikte zwischen unterscheidbaren Kohorten anzusehen sind. Allerdings entstammt dieses Bild einer Situation, in der sich die Schichten oder Klassen mit nur geringen Berührungspunkten aber ohne nennenswerte Mobilitäten gegenüberstanden. Auch wenn die soziale Mobilität nach wie vor gering ist, so kann gegenwärtig, in einer Phase »jenseits von Klasse und Schicht « (Beck 1986) von einer derart fixierten Struktur, in der ja gerade die Tradierung von Kulturformen als Distinktionsmechanismus wirksam ist, kaum noch die Rede sein, auch wenn nicht klar ist, in welcher Weise sich Kontinuitäten als Wandlungen überlagern oder vermischen. Für eine eher konflikthafte und damit aus Gegensätzen gespeiste Entwicklung spricht durchaus die Elias'sche Vermutung, daß der Generationskonflikt im hier gemeinten Sinn, also als Macht- und Besitzkampf zwischen Generationen, ein in der Forschung zwar vernachlässigter, in seiner Wirkung aber den Klassenauseinandersetzungen vergleichbar starker Mechanismus sein könnte.

Als dritte Möglichkeit zwischen diesen beiden Modellen einer Kontinuität auf der einen, eines aus Gegensätzen gespeisten Wandels auf der anderen Seite, könnte eine Entwicklung von Orientierung vermutet werden, bei der jede Generation in einem bestimmten Generationsschicksal (vgl. Mannheim 1976) stehend, ihren originären Be- 
dingungen symbolisch verarbeitet ohne nennenswerten Bezug zu vorausgegangenen oder nachfolgenden Kohorten. Eine Bedingung eines derartigen Generationsschicksals ist jedoch gerade auch der Zugang zu relevanten Positionen. Dieser Zugang zu Einfluß und Besitz ist aber unweigerlich abhängig von den Generationsbeziehungen. Der Gedanke eines typischen Generationsschicksals schließt also notwendig die Bedingungen der Generationsabfolgen ein und diese können als zunehmend konflikthaft und konkurrenzbedingt angesehen werden.

In der Abfolge der kulturellen Orientierungen von Generationen sind dennoch Stabilitäten zu erwarten, da an tradierte Schichtmerkmale gebundene Ungleichheiten noch existieren; es sind jedoch auch Entwicklungen aus Gegensätzen zu vermuten, da die Generationskonkurrenzen mit Sicherheit eine Rolle spielen werden; und es sind auch kontinuierliche Bewegungen zu unterstellen, wenn bestimmte Bedingungen kontinuierlich an Bedeutung gewinnen. Diese unzweifelhafte Überlagerung von Dynamiken und Stabilitäten und die Unsicherheiten wie, d.h. in welchen Wertungen und Präferenzen sie sich ausdrücken, stellen die Schwierigkeiten des Forschungsvorhabens dar. Dazu tritt ein weiteres inhaltliches und methodisches Problem, auf das in ähnlichem Zusammenhang bereits Dollase u.a. (1986) hingewiesen haben, die Unterscheidung von Generations- und Altersorientierungen. Es ist außer bei Massendaten für standardisierte Fragestellungen, wie z.B. in der Forschung zum Wertewandel (Inglehart 1989), äußerst schwierig, zu bestimmen, ob eine bestimmte Äußerung altersoder generationsbedingt ist. Gerade bei Einstellungswandlungen gegenüber dem ästhetisch-symbolischen Bereich innerhalb einer Biographie und zwischen Biographien verschiedener Generationen ist die eindeutige Trennung von Alters-, Generations- oder allgemeinen Zeitphänomen häufig äußerst problematisch und nie ganz befriedigend zu lösen. Werden keine Massendaten produziert - und in der vorliegenden Studie war das ausgeschlossen -, gilt zwar die narrative, biographische Befragung in nicht direktiven Interviews als adäquate Methode, sowohl unbeeinflußte Äußerungen zu erhalten, als auch Einstellungen über die gesamte Biographie hinweg zu ermitteln, und dieser Weg wurde auch in dieser Studie beschritten, dennoch bleiben gerade angesichts des erhobenen Materials, also bei der Durchsicht der Interviewprotokolle, Unsicherheiten bestehen, auch wenn diese Methodik in vergleichbaren Untersuchungen erfolgreich angewendet wurde (vgl. z.B. Bude 1987), bei allen Begrenzungen, die in repräsentativ-quantitativer Hinsicht mit ihr verbunden sein mögen. Da die Generationenzusammenhänge nicht aus quantitativen Daten bestimmt werden konnten, müssen sie aus vorliegender Forschung und auf Plausibilitätsbasis fixiert werden. Unterschieden wurden auf dieser Ebene vier Generationen, die sich ungefähr in Jahrzehntabständen folgen: Um 1930, um 1940, um bzw. kurz nach 1950 und um oder kurz nach 1960 Geborene. 


\section{Die einzelmen Generationen}

\subsection{Die Generation der um 1930 Geborenen}

Daß diese Kohorte eine Generation im Mannheimschen Sinne, also eine Gruppe mit einem gemeinsamen Generationsschicksal bildet, wird kaum bestritten. Als jüngste Publikation hat Bude (1987) die Biographie einiger Angehöriger dieser Jahrgänge um 1930 verfolgt. Als erste Forschungsarbeit hat sich Schelsky (1957) in seiner »Skeptischen Generation « mit ihnen befaßt, wobei er allerdings unterstellt, das die von ihm festgestellten Orientierungen der damals um 20jährigen als typisch für moderne Jugendliche insgesamt anzusehen seien. Als Charakteristika für diese Generation, die den Zusammenbruch des Faschismus z.T. als Flakhelfer, z.T. sogar noch als junge Soldaten erlebte, gelten eben die von Schelsky hervorgehobene Skepsis gegenüber politisch-ideologischen Systemen und frühe, kaum bezweifelte Anforderungen an verantwortungsbewußtes, erwachsenes Handeln. Für den weiblichen Teil mögen ähnliche Kriterien gelten, da zumindest der Zusammenbruch des politischen Systems und die frühen Arbeits- und Verpflichtungsanforderungen in der gleichen Weise erlebt werden. Sogar die Generationsbezeichnung »Flakhelfer-Generation« kann bedingt auch für sie gelten, da in einzelnen Fällen sogar Mädchen oder junge Frauen für diesen Dienst herangezogen wurden.

Die tiefe Verunsicherung durch Zusammenbruch eines politischen Systems gleicht diese Generation aus zum einen durch eine Priorität des Privaten und zwar sowohl im persönlich-intimen Bereich durch die Betonung der Familie, als auch in der beruflichöffentlichen Sphäre durch Präferenzen für organisations- und politikunabhängige, auf individueller Leistung basierende Berufskarrieren, die in kurzen, klar überschaubaren Ausbildungsgängen verfügbar sein sollen.

Obwohl viele Angehörige dieser Generation in den letzten Kriegswochen nicht nur mit dem Zusammenbruch des Faschismus, sonders sehr direkt mit dem Tod, und das heißt auch mit der Wertlosigkeit von Leben und der Zufälligkeit des Überlebens konfrontiert werden, erleben sie jedoch, als Generation und als Individuen notwendig gebraucht zu werden. Es entsteht eine in mehrerer Hinsicht ambivalente Erfahrung, nämlich sowohl »verheizt« als auch, und zwar im Faschismus wie in der neu entstehenden Bundesrepublik, dringend erforderlich, also auch wertvoll zu sein. Daraus scheint sich eine Haltung entwickelt zu haben, die sich charakterisieren läßt als ein Glaube daran, daß der Mensch nur in einer »politikfreien«, ja fast überhistorischen Humanität seinen Wert entfalten könne. Diese Orientierung an Prinzipien bleibt aber sehr entfernt, fast entrückt von den Anforderungen des Alltags, die in individualisierten Leistungsvorstellungen zu bewältigen sind. Auch zur Kirche als Organisationsform führt diese Prinzipiensuche nicht zwangsläufig. Auch Religion wird eher ein Bereich privater Anschauungen und ohne zentrale Bedeutung.

In den kulturellen Orientierungen nun, auf die unsere Untersuchung als Gegenstand zielt, werden diese Erfahrungen und Bedingungen sehr deutlich zum Ausdruck gebracht. In der Kunst und Kulkur werden die dauerhaften Werte gesucht, die schwan- 
kende politische Systeme und historische Bedingungen nicht bieten können. Entsprechend gibt es nach dieser Vorstellung auch eindeutige, in den Objekten liegende und im Zweifelsfall objektivierbare Qualitäten und Werte, also klare Rangfolgen oder Unterscheidungen zwischen dem Guten und Qualitätvollen und dem weniger Guten oder Schlechten. Aber von Bedeutung und Interesse scheinen nur die in überzeitliche Dimensionen gehobenen Spitzenleistungen zu sein. So bezeichnet ein Maler aus dieser Generation die für ihn wichtigen Figuren der Kunstgeschichte als seine »Abgötter«, oder »Cezanne als die Basis von allem«. Eine gleiche Einstellung findet sich jedoch auch und gerade bei denjenigen, die nicht professionell mit Kunst und Kultur umgehen. Es ist ein sehr distanzierter, sehr entfernter, kaum in den Alltag integrierter Umgang, geprägt von einer auf ganz wenige Produkte und Namen begrenzten, diesen aber fast mit Ehrfurcht begegnenden Haltung. Eher Befreiung oder einfach Ablen' kung aus dem Alltag über einen Genuß, der sich auf die ästhetische Dimension bezieht, prägt die Beziehung zu Kunst und Kultur. Studien zur Funktion des Konsums von sog. E-Musik durch ein älteres, bürgerliches Publikum kommen zu ähnlichen Ergebnissen (vgl. Dollase u.a. 1986). Auch Benjamin (1980) hat auf diesen traditionellen Umgang mit Kulturgöttern hingewiesen als kritikloses Wohlgefallen am Bekannten. Es scheint sich für die um 1930 Geborenen darin jedoch mehr auszudrücken, als nur Gewohnheit. Die Sphäre von Kunst und Kultur scheint in allen Unwägbarkeiten, Zusammenbrüchen oder Konflikten politischer Vorgänge das Herausgehobene, der Garant von Humanität und Beständigkeit zu sein. Darin scheint eine Tradition des frühen 19. Jahrhunderts, d.h. der Aufklärung aber auch der Romantik auf, die in dieser Generation der um 1930 Geborenen noch lebendig ist. Eine Besonderheit deutschen Kulturbewußtseins, in Kunst und Kultur eine säkularisierte Religion und, wie es einmal formuliert wurde, eine Suche nach dem fernen Gott zu sehen, ist in Anklängen in dieser Generation noch präsent. Dabei kann die Vorstellung von Kunst in ihrer absoluten ästhetischen Dimension als Versprechen auf eine bessere Menschlichkeit gar nicht einmal als ausschließlich deutsche Tradition gelten, wie das folgende Zitat zeigt, wenn es vielleicht auch kein Zufall ist, daß es von einem Wissenschaftler stammt, der sich gerade mit der mitteleuropäischen Musik des späten 18. Jahrhunderts intensiv befaßt hat:

》Das Mozartsche Vermächtnis, in Kürze, ist die beste Entschuldigung für die Existenz der Menschheit, der wir jemals begegnen werden, und es ist... eine kleine Hoffnung für unser endgültiges Überleben.« (Landon 1988. Der Autor ist 1926 geboren!)

Als Begründung für die Objektivierbarkeit von Kunsturteilen neigen die Angehörigen dieser Generation dazu, auf gleichlautende Urteile von anderen, d.h. von Referenzpersonen hinzuweisen. Dies können Kollegen, Freunde und Bekannte sein. Diese Referenzgruppen sind in hohem Maße schichthomogen. Die dadurch bedingte Gleichheit des Urteils, d.h. des »Kunstgeschmacks«, wird als Begründung für die Objektivität des Urteils gewertet. Die um 1930 Geborenen sind noch nicht von der Erfahrung schichtheterogener sozialer Kontexte geprägt, wie die späteren Generationen. Für diese ist eine solche Objektivierbarkeit aus der Erfahrung divergierender Einschätzung weniger selbstverständlich. Dagegen ist die Alterszusammensetzung 
der Referenzgruppen in der um 1930 geborenen Generation heterogener. Es werden vorwiegend Ältere, also Eltern und Lehrer als entscheidende Personen für kulturelle Eindrücke während der Jugend genannt. Besonders zu Lehrem bzw. Hochschullehrern können dauerhafte Freundschaften entstehen. Entwickeln sich die Beziehungen zu den Eltern eher negativ, findet eine umso intensivere Ausrichtung auf Lehrer statt. Darin liegt ohne Zweifel eine Begründung für die enge Traditionsbindung, die diese Kohorte noch zu prägen scheint. Sie zeigt hier noch die bürgerlichen Einbindungen in altersübergreifende Zusammenhänge und einen geringen Stellenwert von peergroups, also von altershomogenen Szenen und damit auch geringe Neigungen zur Entwicklung einer sog. Jugendkultur. Möglicherweise ist die Generation der um 1930 Geborenen hier sogar wieder zurückhaltender als vorausgegangene Gruppen, bedingt durch negative Erfahrungen mit faschistischen Jugendorganisationen. Auch diese Einbindung in kulturelle Traditionen als Folge vorrangig altersübergreifender Sozialisationszusammenhänge und weniger altershomogener Jugendszenen wird sich in den folgenden Generationen entscheidend ändern.

\subsection{Die Generation der um 1940 Geborenen}

Gemeint ist hier die Generation, von der Teile als $\gg 68 \mathrm{er} \ll$ in die neuere deutsche Geschichte eingegangen sind. Sie sind in der Regel zwischen 1940 und 1945, also zu Beginn der 40er Jahre geboren. Sowohl Selbstdarstellungen wie sozialwissenschaftliche Aufarbeitungen unter der Generationsperspektive werden seit einiger Zeit in wachsendem Umfang publiziert (vgl. z.B. Bude und Kohli, Hrsg. 1989, als ältere Arbeit vgl. Pfeil 1968). Auch hier befassen wir uns nur mit dem bürgerlichen Teil dieser Generation, also nicht mit den Arbeiterjugendlichen der gleichen Geburtsjahrgänge, die bezeichnenderweise durch ihre stärkere Einbindung in altershomogene peer-groups und unbefangenere Öffnung für westliche populäre Kulturprodukte bereits Ende der 50er Jahre in der Rocker-Bewegung den Generationsprotest der 68er vorwegnehmen.

Die Politisierungswelle der späten 60er Jahre als Generationskonflikt zu sehen, ist mittlerweile allgemein üblich geworden, obwohl den Beteiligten diese Perspektive nicht unbedingt eigen war. Vielmehr ging es um tiefgreifende politische Veränderungen, die von den 68ern gefordert wurden, wobei heute, im Rückblick, nachhaltig betont wird, daß wesentlich klarere Vorstellungen dazu bestanden, was abzulehnen sei, als dazu, wie denn die Alternativen aussehen müßten, ein deutliches Indiz für einen Generationskonflikt.

Als eine gemeinsame Grunderfahrung der in den ersten Jahren der 40er Jahre Geborenen wird in den genannten Selbstzeugnissen wiederholt die Schulzeit in den lähmenden, restaurativen 50er Jahren genannt. Dazu kommen erlebte Widersprüche zwischen den politischen und Bildungsinhalten, wie sie die zentralen Sozialisationsinstanzen in der Bundesrepublik intern vermitteln und den Einschätzungen solcher Inhalte im Ausland, das von dieser Generation durch die Öffnung der Bundesrepublik sowohl durch Reisen, Schüler- und Studentenaufenthalte als auch durch Kulturim- 
porte erlebt wird. Ähnliche Widersprüche werden aber auch wahrgenommen zwischen nominellen Ansprüchen in der Bundesrepublik, z.B. in bezug auf die Rolle als Kulturstaat oder als freiheitliche, dynamische Demokratie und der Realisierung solcher Ansprüche durch ein hochgradig überaltertes, in den Augen der Jüngeren nicht gerade höchste kulturelle Bildung ausstrahlendes Personal. So empfindet Häußermann den damaligen Bundespräsidenten Heinrich Lübke als »kaum der deutschen Sprache mächtig « (1989:47) und einer der Interviewten nennt die Diskrepanz zwischen der grundsätzlich garantierten Pressefreiheit und dem faktischen Monopol des Springerkonzerns als einen solchen Widerspruch zwischen Anspruch und Realität in der damaligen Bundesrepublik. Als zentrale Erfahrung vermitteln jedoch alle Zeugnisse das Gefühl der Machtlosigkeit gegenüber oder des Ausgeschlossenseins von Entscheidungsprozessen. Selbst milde, gemäßigte Veränderungs- oder Verbesserungsvorschläge werden entweder nicht zur Kenntnis genommen oder rufen undifferenzierte, von Gewalt und Autorität getragene Ablehnungen hervor und dies angesichts nomineller Aufforderungen zu politischer Teilhabe an den Institutionen der Bundesrepublik. Dies ist für Elias (1989) eine typische Konstellation, eine typische Atmosphäre für sich fast zwangsläufig eskalierende Generationskonflikte, wie sie dann im Verlauf der 70er Jahre in der Form des neuen deutschen Terrorismus auch eingetreten sind. Ohne daß es möglich wäre, die Bedingungen dieser oder der anderen Generation an dieser Stelle erschöpfend darzustellen, sind die Ausprägungen kultureller Orientierungen in dieser Generation der $68 \mathrm{er}$ von erstaunlicher Konsequenz sowohl in bezug auf die Kontinuitäten, als auch auf die Brüche zur vorhergehenden Generation der um 1930 Geborenen, obwohl es nicht explizit diese Kohorte ist, gegen die sich der Protest der 68er richtet, bzw. mit der sie um Positionen konkurriert. In der hohen Wertschätzung von Kultur stehen auch die um 1940 Geborenen ganz in der bürgerlich-deutschen Tradition. Sie steigern sie sogar im Vergleich mit den um 1930 Geborenen. Im Unterschied und in deutlicher Unterscheidung von dieser Generation soll Kunst und Kultur jedoch aus dem quasi religiösen Nimbus, aus der überzeitlichen Dimension des Schönen befreit werden. Von Bedeutung für die Generation der 40er Jahre ist der auf konkrete Zeitphänomene der Gegenwart oder einer definierten historischen Epoche bezogene Inhalt eines Kunstproduktes. Mit der ästhetischen Seite, die sie als »Kunstgenuß bezeichnen, haben sie dagegen größte Probleme, auch wenn die vehementen Ablehnungen dieser Aspekte im Verlauf des weiteren Lebens etwas relativiert werden. Dem »Bildungsbürger «, wie sie ihn verstehen, und der sich als distanzierter und genießender, in der Kunst Ablenkung oder Freude suchender Laie darstellt, setzen sie den professionellen Analytiker entgegen, der Kultur als Beruf betreibt und aus Kunstprodukten Informationen für seine Arbeit zieht. Nicht umsonst wird von der Generation der um 1940 Geborenen wiederholt Martin Walser als hochgeschätzter Autor genannt, dessen Bücher ja durchaus als Soziologie im literarischen Gewand angesehen werden können. In der vorhergehenden Generation fällt dieser Autorenname nicht einmal. Es werden dagegen nur Klassiker, also die des 18./19. Jahrhunderts oder der Moderne genannt, in der Literatur also z.B. Joyce und Proust, die wiederum bei den Interviewten aus der Generation der um 1940 
Geborenen nicht erwähnt werden. Neben Walser sind es häufig die großen amerikanischen Realisten, die eine Rolle spielen, deren sozialanalytische Dimension nicht zu übersehen ist oder die Romanciers der zweiten Hälfte des 19. Jahrhunderts, gelesen unter der Perspektive, die ein Befragter so formuliert: »Um das 19. Jahrhundert zu verstehen, genügt es nicht, Nipperdey oder Wehler zu lesen, dazu gehören die Romane. «Nach Bourdieuschen Kategorien setzt die Generation der um 1940 Geborenen in der Figur des aufgeklärten, engagierten Gelehrten eine Gegenposition zur 1930er Generation, die sich eher am $»$ Unternehmer « orientiert, also an einer Figur, die Kultur ohne große Kenntnisse bestenfalls als Hobby und Sammelei betreibt. Die kleinbürgerliche Variante dieses »Unternehmers «, mit wenig Kenntnis und etwas Besitz wird zum absoluten Feindbild. Darin wird dann auch die eigene kulturelle Leistung der 68er-Zeit gesehen, die ein Interviewter so bezeichnet: »Die kleinbürgerliche Horterei war zu Ende«. Mit der analytischen und professionellen Perspektive wird auch die Neigung, »absolute Werte in einzelnen Werken zu sehen, reduziert. Unterschiedlichste Produkte können als Informationsquelle brauchbar sein und alle erhalten einen relativen Wert nur aus dieser Dimension. Sogar der Weg zur neuen angelsächsischen Pop-Musik wird auf diese Weise gefunden.

Damit stellt sich der Generationskonflikt, den die 68er artikulieren, dar als Entfaltung und vehemente Aufwertung intellektueller, kulturell-symbolisch geprägter Karrieregänge in Beamten- oder Angestelltenpositionen ohne materielles Kapital oder Vermögen und als Abwertung freiberuflicher oder unternehmerischer Karrieren, wie sie die Anfangsphase der Bundesrepublik geprägt haben. Die um 1940 Geborenen vollziehen damit die in den 60er Jahren notwendige Modernisierungsphase der BRD in Richtung auf eine Dienstleistungsgesellschaft, eine Modernisierung, die sich an Bewertungskonkurrenzen unterschiedlicher Karrierewege deutlich macht. $\mathrm{Daß}$ diese Neubewertung intellektuell-wissenschaftlicher Dienstleistungsberufe sich als außerordentlich erfolgreich erweist für die 1940er Generation, obwohl sie möglicherweise als Ausweich- oder Umwegstrategie vor den blockierten Institutionen der 50er JahreBundesrepublik angelegt war, ist eine der Kritiken, die den 68ern von nachfolgenden Generationen später immer wieder entgegengehalten wird. Für die um 1940 Geborenen gilt jedoch tatsächlich, was eine Befragte, die nach eigener glaubhafter Aussage ganz und gar nicht zu den »echten« 68ern gehört hat, unbewußt doppelsinnig formuliert: »Man ist ja mit Kultur groß geworden «.

Diese Interpretation des 68er-Generationskonflikts als Konkurrenzen verschiedener Karriereleitern, also weder als Konkurrenz in einem Karrieresystem, noch als Entwicklung von Positionen und Anerkennung ohne Karrieren, eine Sicht, die in den kulturellen Orientierungen sehr deutlich zum Ausdruck kommt, erklärt auch ansatzweise, warum die vehement als antiautoritär auftretende Studentenbewegung der $68 \mathrm{er}$ Jahre zwar in vielen symbolischen Bereichen eine $»$ Kulturrevolution« mit langfristigen Folgen in Gang setzen konnte, autoritäre und konkurrenzgeprägte Beziehungen jedoch massiv reproduzierte, außer, wie glaubhaft behauptet wird, in Teilen der Frauen- und der Kinderladenbewegung (vgl. Hopf 1989).

Kennzeichnend für die Referenzgruppen dieser Generation wird die deutlich ausge- 
prägte Altershomogenität, die sich z.B. in den Wohngemeinschaften ausdrückt und die Tradierungstendenzen von kulturellen Orientierungen im Gegensatz zur vorhergehenden Generation aufzubrechen beginnt. Auf der anderen Seite ist jedoch die Schichthomogenität auch in dieser Generation noch weitgehend erhalten, so daß die Neigung, aus gleichen Urteilen im Referenzkontext auf objektivierbare Werturteile zu schließen, noch besteht. Bestimmend wird für diese Generation ein intellektuelles Milieu an den Hochschulen und in den Großstädten, das für die Vorhergehenden so nicht bestanden hatte und auf das sich die Befragten nachdrücklich beziehen.

\subsection{Die Generation der um 1950 Geborenen}

In zahlreichen Jugendstudien, die in den 70er und frühen 80er Jahren entstehen, bildet diese Generation den Untersuchungsgegenstand. Meist liegt jedoch die Betonung dabei auf dem Alter, nicht auf der Generationszugehörigkeit, d.h. auf dem spezifischen Generationsschicksal dieser Jahrgänge. (Eine Ausnahme stellt die Untersuchung von Baethge u.a. 1987 zum - vermeintlichen - Wertewandel bei Lehrlingen und Schülern dar.) Für die hier thematisierten Teile dieser Generation mit universitärer Ausbildung gelten in eigenartiger Weise umgekehrte Bedingungen, wie für die vorhergehende Kohorte der um 1940 Geborenen, so daß sie wiederum in ganz anderer Weise als diese Kontinuitäten und Brüche ihrer Orientierungen entwickeln.

Die Generation der um 1950 Geborenen erlebt im Unterschied zur vorhergehenden Gruppe in der Schulzeit keine Verhärtung, aus der sie sich dann in der Universität unter dem Gefühl des selbst bewerkstelligten Aufbruchs befreit, sondern wird ganz im Gegenteil von der Liberalisierung der 60er Jahre und von der Reform und Öffnung des Bildungssystems in der eigenen Schulzeit - passiv - erfaßt. Da sie sich notwendig mit der Jugendorientierung der Studentenbewegung identifizieren muß, verstehen sich viele selbst noch als 68er und finden in dieser Vorgängergeneration und ihren Inhalten ihre Orientierungspunkte. In der Hochschule wird diese Atmosphäre fortgesetzt, die ein Befragter so bezeichnet: »Für mich war die Universität eine Pralinenschachtel«. Dies gilt jedoch häufig eher für die Lehrinhalte und die Beziehung zum Personal, nicht für die Situation in politischen oder hochschulpolitischen Gruppierungen. Entscheidender jedoch als dieser Aspekt ist die in der Bildungsforschung intensiv behandelte Tatsache, daß nicht nur die formalen Freiheiten der Ausbildungszeit, sondern auch deren Inhalte und vor allem die Zertifikate in der Berufssituation abgewertet werden (vgl. dazu z.B. Rolff 1980) und zwar in jeder der beiden prinzipiellen Karriereleitern, von denen oben die Rede war. Da eine dritte Alternative nicht existiert, reagieren in dieser Generation viele mit der Abwertung von Karrieren insgesamt oder mit aggressiver Konfrontation zum sog. »System«. Während also für die 40er-Jahre-Generation durchaus gilt, daß sie mit oder durch Kultur groß wird, kann man zugespitzt sagen, daß die folgende Kohorte der um 1950 Geborenen mit Kultur klein bleibt. Wie in keiner Generation vorher oder nachher beginnt diese Gruppe, Jugend in die Länge zu ziehen. Entsprechend der Hochbewertung von Kultur in den Ausbildungsinstituten und orientiert am Vorbild der 40er-Jahre-Generation, der man 
sich, wie gesagt, fast zugehörig fühlt, bleibt dieser Bereich zentral für Umwegkarrieren, die jedoch nicht mehr als instutionelle, sondern als freie Aufstiege oder als Hoffnungen auf persönliche Durchbriiche gesehen werden. Der Ausbildungssituation entsprechend entsteht eine Aufweichung von Professionalitätsvorstellungen, die sich kulturell in einer Betonung von Gefühlsmomenten in Produktionen und in Äußerung gegen den Rationalitäts- und Objektivitätsanspruch der Vorgängergeneration wendet. In den kulturellen Biographien wird das deutlich an einer spezifischen, engen »Anklammerung « an frühe, fast kindliche Kulturprodukte, an eine spezifische Verbindung sehr ernsthafter mit spielerischen Elementen im Umgang mit Kulturprodukten, an einer ausgesprochen hohen Bewertung emotionsgetragener und - scheinbar - eskapistischer Werke und an der Entwicklung eng persönlichkeitsbezogener, von Erinnerung geprägter, hoch libidinös besetzter Sammlungen von scheinbar bedeutungslosen, symbolisch aufgeladenen Gegenständen. Teilweise werden diese Vorstellungswelten extrem lange aufrecht erhalten, so daß z.B. ein Befragter, zum Zeitpunkt des Interviews knapp 40 Jahre alt, der niemals eine professionelle Ausbildung im Filmwesen durchlaufen und bislang erst einen Experimentalfilm gedreht hat, noch immer davon ausgeht, bald seinen ersten abendfüllenden Spielfilm zu verwirklichen.

Es wird jedoch auch eine gegensätzliche Haltung und Biographie-Konstruktion erkennbar. Während die 40er-Jahre-Generation zwar die Unternehmer-Karriere abund die Wissenschaftler- und Angestelltenkarriere auf kultureller Basis aufwertet, diese Aufwertung jedoch in teilweise sehr traditionellen Formen von Arbeitsethos vollzieht, also zum »Verzicht auf Leben« ungewollt bereit ist - auch wenn sich gefühlte Defizite daraus für ihr ganzes Leben einstellen - versucht die 50er-Jahre-Generation tatsächlich zu leben. In extensiven Reisen häufig über mehrere Monate wird dies sehr deutlich. Die spielerisch-auswählende Haltung, die im Umgang z.B. mit der Universität zitiert wurde, scheint in hoher Experimentierfreudigkeit auch gegenüber anderen, vielleicht gegenüber allen Aspekten des Lebens eingenommen zu werden. Und dennoch signalisieren die Befragten auch ein Leiden an unbewältigter Überfülle der Alternativen. Sie erwecken den Eindruck, von permanenten Entscheidungsanforderungen und Wahlvorgängen, sei es zwischen Angeboten des Warenmarktes, sei es zwischen Alternativen der Lebensplanung, überfordert zu sein. Das führt, da durch unvergleichlich verstopfte Karriereleitern keine Entscheidung zu einer befriedigenden Positionierung wird, zu einer deutlichen Psychologisierung der eigenen Biographie, die als Entlastung für Mißerfolge eingesetzt wird, aber auch zu radikalen biographischen Brüchen noch im fortgeschrittenen Alter, mit denen auf Kultur begründete, nur bedingt erfolgreiche Karrieren abgebrochen werden. Gerade Angehörige dieser Kohorte, die in der ersten Familiengeneration Akademiker geworden sind - angesichts der Bildungsexpansion eine relevante Zahl -, scheinen eine Tendenz zu entwickeln, schlagartig die Lebensform der Eltern wieder aufzunehmen, also z.B. den elterlichen Betrieb oder Laden zu übernehmen oder etwas ähnliches neu zu begründen. Aus diesem Personenkreis speisen sich u.a. die Betreiber von Alternativ-Läden in den Großstädten aber auch Kleinunternehmertum in neuen Dienstleistungen und Medien. In den kulturellen Orientierungen wird wieder, wie schon bei der Vorgänger- 
generation, die widersprüchliche Bindung an die nun ca. 10 Jahren Älteren erkennbar. Die Vorstellung, daß Kultur und Kunst zur Verbesserung der Welt beitragen könne, wird (noch) nicht aufgegeben. Ihre Leistung liegt aber nicht in der Analyse und Information, sondern in der Moral, die das Werk transportiert, in der Gefühlswahrheit, in ihrem Beitrag zur Selbstfindung des Individiums, d.h. in den Identifikationschancen. Dies ist eine Anforderung, die von einem aufklärerisch-kritischen Kunstverständnis eher als kitschig empfunden wäre; und es ist häufig gerade diese Ebene des nach älteren Kriterien kitschig-banalen, die auf diese Generation besondere Anziehungskraft auszuüben scheint.

Bei einem solchen Befund liegt es natürlich nahe, die herausgearbeiteten Besonderheiten nicht für generations- sondern für schichtspezifisch zu halten, denn z.B. nach dem Bordieuschen Geschmacksmodell bezeichnet die Betonung inhaltlicher Dimensionen von Kulturprodukten oder gar die Sicht dieser Inhalte unter moralischen Kriterien eine kleinbürgerliche, eventuell sogar proletarische Position gegenüber der bürgerlichen, die eine objektimmanente Ästhetik als Qualitätskriterium benennt (Bourdieu u.a. 1981). Zwar haben alle die Befragten, auf die sich diese Aussagen beziehen, ein Hochschulstudium, aber dennoch können Schichtdifferenzen hier eine Rolle spielen. Durch die Bildungsreform sind Einstellungen der unteren Angestellten- und Arbeiterschaft in die Hochschulpopulation diffundiert. Die Einstellungsänderungen der Hochschulabsolventen im Generationsablauf können also auch ein Hinweis auf die Wandlungen der Schichtstruktur dieser Gruppe sein. Auf der Basis der verwendeten Methodik und der geringen Fallzahlen lassen sich sichere Aussagen hierzu nicht treffen. Es liegt aber durchaus nahe, daß die kulturellen Orientierungen der 50er- und 60er-Jahre-Generation von traditionell kleinbürgerlichen Verhaltensweisen geprägt sind, da in die Schul- und Ausbildungszeit dieser Kohorten die Bildungsreform fällt. Welche Differenzierungen nun wiederum in dieser Population vorliegen zwischen tradiertem und neuem Akademikertum, zwischen neuer humanwissenschaftlicher und neuer technischer bzw. zwischen alter und neuer Intelligenz, läßt sich auf dem bisher erreichten Forschungsstand nicht sagen. Dazu müßten die Tiefeninterviews durch umfangreiche Massendaten ergänzt werden.

Für die Generation der um 1950 Geborenen gelten in bezug auf die Referenzgruppen andere Bedingungen als für die vorhergehenden Kohorten. In der Altershomogenität dieser Bezüge ähneln sie der um ca. 10 Jahre älteren Generation und unterscheiden sich mit dieser von den um 1930 Geborenen. In der Schichtheterogenität, die sich durch die Bildungsreform und andere Mobilitätsvorgänge bereits sehr früh bei diesen um 1950 Geborenen einstellt, unterscheiden sie sich von beiden vorausgegangenen Generationen. Mit der Folge dieser Bedingung, und zwar mit der Tendenz, Objektivierbarkeiten in den Kunst- und Werturteilen zweifelnd gegenüberzustehen und nur individuelle Wertschätzungen und Prioritäten für möglich zu halten, nähern sie sich der letzten Generation, der um 1960 Geborenen. Es beginnt sich hier also eine Orientierung durchzusetzen, die sich sowohl von Tradierungen wie von Objektivierungsversuchen des Geschmacksurteils löst, eine Haltung, die bei der folgenden Generation sehr deutlich artikuliert wird. 


\subsection{Die Generation der um 1960 Geborenen}

Je jünger die Generationen zum Zeitpunkt der Befragung sind, um so schwieriger ist nicht nur die Durchführung eines biographischen Interviews, das ja Biographie und deren Reflexion voraussetzt, sondern auch die Fixierung eines Generationsschicksals und der typischen Repräsentanten eines solchen Bedingungskomplexes. Das gilt besonders, wenn die überschaubare Lebensspanne nicht von gravierenden Großereignissen begleitet und eventuell geprägt ist. Dennoch lassen sich auch für die um 1960 Geborenen, die Ende der 60er Jahre und in den 70er Jahren die Schule und in den frühen 80er Jahren die Universitäten aufsuchen, Beeinflussungen vermuten, die in den Interviews durchaus ihren Niederschlag finden. Zum einen repräsentiert diese Kohorte nochmal in einer Wiederholungswelle zu den um 1940 Geborenen eine Reihe geburtenstarker Jahrgänge. Sie erleben also die Bildungseinrichtungen in ständiger Überfüllung. Das wird durch die ausgeweitete Bildungsteilnahme noch gesteigert. Insofern findet die Bildungsreform für diese Jahrgänge durchaus eine Fortsetzung. Die Liberalisierungswelle jedoch, die für die vorhergehende Generation der um 1950 Geborenen die Schul- und Universitätszeit geprägt hatte, ist abgeebbt. Die Konkurrenz des Arbeitsmarktes reicht jetzt gravierend in die Ausbildungseinrichtungen hinein. Die Überfüllung und Verstopfung der kulturgeprägten Ausbildungs- und Karrierewege zwingt zur Orientierung an neuen Berufsmustern in Naturwissenschaften und neuen Technologien. Eher konservative und kleinbürgerliche Teile dieser 50er JahreGeneration behalten die Orientierung an den kulturellen Karrieren - ihrer Eltern? bei. Sonst erfolgt eher eine Abwertung dieser Perspektiven außer in einigen expliziten Kunstberufen, die an der Stelle überschaubarer aber besetzter Karrieren eine erhebliche Bedeutung als Um- oder Auswege erhalten. So geht zwar der Andrang auf sozialund geisteswissenschaftliche Ausbildungen mit klaren Berufsbildern zurück und es erfolgt auch eine Abwertung dieser Perspektive, im Bereich der Kunst jedoch mit ihren offenen Berufsperspektiven und unkalkulierbaren Personalbedarfen aber auch mit ihren medienvermittelten Selbstverwirklichungsversprechen entsteht ein dramatisch ansteigendes berufliches Interesse. Benjamin (1980) hat diese zweite Seite der Selbstverwirklichungsillusion der medienvermittelten Kunstformen bereits in den 30er Jahren gesehen. Dabei soll hier nicht auf den Streit eingegangen werden, ob es sich bei diesen Selbstverwirklichungswünschen um einen Wertewandel handelt, der in krisenhafter Weise Pflicht- und Akzeptanzwerte aufweicht oder eher um eine Reaktion auf verengte Möglichkeiten der Selbstverwirklichung (vgl. dazu Baethge u.a. 1987). Sicher ist, daß die Medien und der Markt von Kulturgütern und ihre Verbreitung die - meist unrealistischen - Hoffnungen auf diese Bereiche als Berufsziele nachhaltig fördert. So scheinen sich in der 60er-Jahre-Generation ausgesprochene Pragmatiker der Biographieplanung auf der einen und Selbstverwirklicher auf der anderen Seite gegenüber zu stehen. Beide sind jedoch von schulischen und universitären Konkurrenzerfahrungen geprägt. Sie kennen den geringen Nutzwert von Zertifikaten, wenn diese nicht brillant oder in irgend einer Weise privat ausgebaut oder ergänzt werden. 
Diese Erfahrung, in der Überfülle und Konkurrenz unter Profilierungsdruck zu stehen, scheint die eine Seite zu sein. Die andere ist wohl die des Entscheidungszwanges, des Wählens unter Alternativen unter hohem Risiko, d.h. angesichts von Konkurrenz falsch zu wählen. Während die vorhergehende Generation den Eindruck vermittelt, bei der Wahl von Lebensalternativen und d.h. mit Brüchen oder Wechseln in der Biographie relativ leicht und ohne das Gefühl der Bedrohung durch Chancenverlust umzugehen, ist die nachfolgende Generation hier wesentlich zurückhaltender. Die Notwendigkeit, sich zu entscheiden, und zwar relativ früh in der Biographie, wenn keine Nachteile in der Konkurrenz um Positionen entstehen sollen, scheint zumindest dem Teil der Pragmatiker, die diese Generation entscheidend prägen, sehr viel deutlicher, als der vorausgegangenen Kohorte.

Ähnlich sicher wie diese biographischen Auswahlvorgänge wirken diejenigen von Waren und damit auch im Bereich der symbolischen Güter. Die in den 60er Jahren Geborenen sind möglicherweise die erste bundesdeutsche Generation, die angesichts eines vollentwickelten Warenmarktes aufwächst und die von frühester Kindheit an zur Entwicklung von »Konsumentensouveränität« gezwungen ist. Neben der Überfüllung der Ausbildungsinstitutionen, den damit verbundenen Schwierigkeiten von Berufseinstiegen in herkömmlichen Karrieren und den Reaktionen darauf durch Pragmatismus und Konkurrenzhaltungen macht diese Konfrontation mit dem Warenmarkt unübersehbar ein entscheidenes Moment des Generationsschicksals dieser Gruppe aus, so undramatisch es auch auf den ersten Blick scheinen mag.

$\mathrm{Da}$ auch diese Generation wie die vorhergehende aber vielleicht noch in gesteigertem Maße in altershomogenen aber schichtheterogenen Referenzgruppen aufwächst, ist sie angesichts des entfalteten Warenmarktes, der sie als Konsumenten sucht, in neuer Intensität mit Auswahlentscheidungen zwischen Gütern konfrontiert, bei denen jeder Einzelne weitgehend auf sich gestellt ist und wo Entscheidungen in den Auseinandersetzungen mit Altersgleichen als persönliche Ausdrucksformen gefunden werden müssen. In den Äußerungen zu Kunst und Kultur kommen diese Bedingungen mit Schärfe zum Ausdruck. So wird die Möglichkeit der Objektivierung von Qualitäten oder Werten mit Nachdruck abgelehnt und zwar gerade auch von professionellen Künstlern. Dies geschieht konsequenterweise mit dem umgekehrten Argument, wie es die Generation der um 1930 Geborenen als Begründung für eindeutige Qualitätskriterien abgab. War es dort die Gleichheit des Urteils in homogenen Referenzgruppen, so ist es bei den um 1960 Geborenen die Erfahrung der ständig ungleichen, nicht übereinstimmenden Urteile in den heterogenen Kontexten, wie sie für diese jüngeren Jahrgänge zur Selbstverständlichkeit geworden sind. Es wird in den Interviews immer wieder betont, daß es eben dieses eindeutig Beste nicht gäbe, daß aus unterschiedlichen Genres Gutes und weniger Gutes vorliegen könne, und daß diese Urteile auch nur von jedem einzelnen gefällt werden könnten; und darauf wird mit Nachdruck insistiert, daß der Weg zu Bewertungen, die natürlich jeder finden muß, selbständig und nicht in der Orientierung an anderen zu bewältigen ist. Selbst wenn sich das Urteil am Ende sichtlich mit dem vieler anderer deckt, wird auf der Eigenständigkeit, auf der eigenen Entdeckerleistung oder Urteilsbildung beharrt. Auch diese Urteilsbildung 
wird als höchst subjektiver Vorgang gesehen. In der Formulierung: »Das erzählt mir eine Geschichte «, zu ergänzen um... die nur ich verstehe, die nur mich betrifft... kommt das zum Ausdruck. Auch andere mögen das gleiche Werk schätzen, es »erzählt« ihnen dann aber mit Sicherheit etwas anderes, wissen kann man das nicht, und es spielt in dieser Sicht anscheinend auch keine Rolle.

Es ist offensichtlich, daß eine solche Wertungskategorie denkbar weit entfernt ist von den auf ästhetischen Kategorien basierenden, allgemeinen Humanisierungsgedanken der ersten aber auch von der inhaltlich-analytischen Aufklärungsanforderung der zweiten hier behandelten Generation. Es mögen Ähnlichkeiten zur Generation der um 1950 Geborenen bestehen, aber deren Neigung zur Psychologisierung fehlt. Wenn die 50er Jahre-Gruppe dazu neigt, ihre Präferenzen selbst als Folge eines Sozialisierungsvorganges zu erklären, bestehen die Jüngeren, die um 1960 Geborenen, auf den autonom durchgeführten Wahlentscheidungen, die sie selbständig und gegen Konflikte vollzog̉en haben.

Natürlich liegt es nahe, diese Selbstsicht weniger generationsspezifisch als vielmehr alterssspezifisch zu sehen. Es könnte die Lebensphase - Ende Zwanzig - sein, die solche Selbsteinschätzungen nahelegt und die sich möglicherweise in zehn oder 20 Jahren der der jetzt entsprechend älteren angepaßt haben könnte. Und doch spricht der entschiedene und dennoch spielerische, der breit angelegte und von Autoritäten freie Wahlvorgang, der zu den persönlichen Wertschätzungen führt, dafür, daß die letzte Generation etwas realisiert, was bei der vorhergehenden eventuell in diesem Lebensabschnitt auch angelegt war, aber nicht entfaltet wurde: Die Anforderung an Kunst und Kultur, den eigenen Wünschen und momentanen Bedürfnissen zu genügen. Nicht ästhetische Verklärung, nicht Aufklärung oder Moral wird erwartet, sondern die »Lieferung eines ehrlichen Produktes «, »Authentizität«, dies schwer aufzulösende Zauberwort, in das gerade die jüngsten Befragten ihre Anforderung kleiden. Diese Kategorie des Authentischen als Qualitätsmerkmal bezeichnet eine Stimmigkeit, eine Richtigkeit von Werk, Produkt, Medium und Vermittlungskontext, wie sie so von keiner vorhergehenden Generation beschrieben wird. Sich von anderen Kriterien bei der Bewertung von Kultur freizumachen, also z.B. vom Bildungsanspruch, von der ästhetischen Qualität, von der Originalität oder Moralität, kennzeichnet geradezu einige Bildungsbiographien in der Generation der um 1960 Geborenen.

In der Suche nach dem Authentischen, das der eigenen Befindlichkeit entspricht und »eine Geschichte erzählt «, entwickelt diese letzte Generation einen erstaunlichen Eklektizismus, in dem sie die vorhergehenden Jahrgänge weit übertrifft und der von Befragten der 40er-Jahre-Generation auch mißbilligend als »postmoderne Beliebigkeit «kritisiert wird. Das zeigt, daß die Abwertung der Prinzipien, die für diese Gruppe galten, durch die Jüngeren gelingt. Was gewählt wird, ist durchaus nicht beliebig, auch wenn scheinbar disparate Dinge verbunden werden. Zum einen drückt sich hier die Wahl als Wahl, also als persönliche Entscheidung aus, was die Form der Montage bzw. der aus Jugendstilanalysen bekannten Bricolage annehmen kann. Zum anderen folgen die Auswahlen den Anforderungen, denen diese Generation unterliegt: In sehr heterogenen, konkurrenzgeprägten Milieus durch ästhetische Symbole Zugehörig- 
keiten und damit Homogenitäten von sozialen Kontexten herzustellen oder, gleichfalls als Entlastung zur Konkurrenz, Großveranstaltungen mit Massencharakter aufzusuchen, in denen sich diese Masse selbst erlebt, was gegenwärtig fast nur noch in der Kultur geschieht. Daß Großveranstaltungen diese Funktion haben, war sowohl Goethe angesichts der Arena von Verona (vgl. Eintragung im Tagebuch zur italienischen Reise vom 16. September) als auch Benjamin (1980) angesichts des Films aufgefallen.

Es ist nicht möglich, in diesem begrenzten Beitrag auf alle Facetten des kulturellen Verhaltens aller Generationen einzugehen. Für die letzte, die um 1960 Geborenen, drängt sich jedoch auf, daß sie gegenüber Kunst und Kultur eine ausgesprochene Dienstleistungsanforderung entwickelt und damit sowohl den eigenen Bedingungen der Konfrontation mit einem entwickelten Warenmarkt entspricht, als auch eine Gegenposition zu den vorhergehenden Generationen einnimmt, die diese Haltung als bestenfalls beliebig, eher aber noch als Auslieferung an die Marktmanipulationen der Kulturindustrie kritisiert. Genau von der dahinter stehenden Anspruchshaltung, die Kultur als notwendig kritisch sehen will, und die die Älteren nachhaltig prägt, scheint sich die jüngste Generation in gezielter Distanzierung abzusetzen.

\section{Zusammenfassung}

Aufeinanderfolgende Generationen tradieren nicht nur Normen und Werte, sondern stehen zunehmend in Konkurrenzbeziehungen um Positionen und Einkommen. Diese Konkurrenzen führen zu Distanzierungen zwischen Generationen, die in relativ kurzen Zeiträumen, also weniger als den 30 Jahren umfassenden biologischen Generationsspannen einander ablösen. In sogenannten kulturellen Orientierungen, also in Einstellungen und Wertungen gegenüber dem ästhetisch-symbolischen Bereich, kommen die Konkurrenzbeziehungen zu vorhergehenden und möglicherweise auch nachfolgenden Generationen zum Ausdruck. So entwickelt jede Generation einen spezifischen Generationshabitus, zu dessen Ausprägung neben anderen Faktoren die Beziehungen der Generationen untereinander beitragen.

Beim Vergleich der vier Generationen der bundesdeutschen Bevölkerung, den Anfang 1930, Anfang 1940, um 1950 und um 1960 Geborenen, wird eine Entwicklung erkennbar, in der sich Kontinuitäten und Gegensätze überlagern und zu generationsspezifischen Ausprägungen führen. In Stichworten lassen sich die kulturellen Orientierungen oder das Kulturverständnis jeder Generation folgendermaßen charakterisieren:

- Die um 1930 Geborenen: Kunst und Kultur sehen sie als eher entfernte, säkularisierte Religion, als Sphäre der überzeitlichen Werte und Garant von Humanität; daneben ist die Beziehung des Einzelnen zu diesem Bereich von Gütern und Werten eher von Besitzkategorien geprägt.

- Die um 1940 Geborenen: Kunst und Kultur gelten ihnen als Instrument realhistorischer Analyse und Information, dem sie mit dem Anspruch professioneller Kenntnisse, nicht mit Besitzkategorien begegnen. 
- Die um 1950 Geborenen: Kunst und Kultur werden ihnen zur Sphäre individueller Gefühlsentfaltung und biographischer Selbstvergewisserung; Relativierung von Wertkategorien im Bereich der Kunst und Kultur zugunsten spielerisch-kontemplativer Haltungen.

- Die um 1960 Geborenen: Kunst und Kultur werden gesehen als Bereich des Warenmarktes neben anderen, mit der Aufforderung und Möglichkeit freier, persönlich geprägter, aber an Szene-Diskurse angebundener Auswahlentscheidungen, die explizit als eigene deklariert werden müssen und eine Objektivierung von Werturteilen ausschließen.

Insgesamt bedeutet die Entwicklung, die durch diese vier Generationen markiert wird, das Ende eines »deutschen kulturellen Sonderweges «, der sich durch eine auratische Überschätzung von Kultur und Kunst als das »Bessere «, das »Eigentliche « oder das »Richtige «, aber nie erreichbare Leben auszeichnete. Eingesetzt hat statt dessen eine Verwestlichung der Kultur bzw. des Kultur- und Kunstverständnisses, in dem Kunst und Kultur Dienstleistung und ein Warensektor neben anderen Märkten wird, dadurch aber in der Alltagsbedeutung als Kommunikationsgegenstand und Symbolbereich für Distanzierungen und Differenzierungen eher gewinnt.

\section{Literatur}

Baethge, Martin u.a. (1987): Jugend und Krise. Eine empirische Untersuchung zur Bedeutung von krisenhaften Arbeitsmarktentwicklungen für Arbeitsbewußtsein, Arbeitsverhalten und Interessenorientierung von Jugendlichen/jungen Erwachsenen, Göttingen

Beck, Ulrich (1986): Risikogesellschaft. Auf dem Weg in eine andere Moderne, Frankfurt a.M.

Benjamin, Walter (1980) (zuerst 1936): Das Kunstwerk im Zeitalter seiner technischen Reproduzierbarkeit. Gesammelte Schriften I.2, Frankfurt a.M.

Bourdieu, Pierre u.a. (1982): Die feinen Unterschiede, Frankfurt a.M.

Bourdieu, Pierre (1981): Eine illegale Kunst. Die sozialen Gebrauchsweisen der Photographie, Frankfurt/ a.M.

Bude, Heinz (1987): Deutsche Karrieren, Frankfurt a.M.

Bude, Heinz und Martin Kohli (Hrsg.) (1989): Radikalisierte Aufklärung. Studentenbewegung und Soziologie in Berlin 1965 bis 1970, Weinheim/München

Dollase, Rainer u.a. (1986): Demoskopie im Konzertsaal, Mainz-London-New York-Tokyo

Elias, Norbert (1989): Studien über die Deutschen. Machtkämpfe und Habitusentwicklungen im 19. und 20. Jahrhundert, Frankfurt a.M.

Häußermann, Hartmut (1989): Das Berliner Milieu und die Stadtforschung. In: Bude, Heinz und Martin Kohli (Hrsg.): Radikalisierte Aufklärung. Studentenbewegung und Soziologie in Berlin 1965 bis 1970 , Weinheim/München, S. 43-70

Hopf, Christel (1989): Das Faschismusthema in der Studentenbewegung und in der Soziologie. In: Bude, Heinz und Martin Kohli (Hrsg.): Studentenbewegung und Soziologie in Berlin 1965 bis 1970, Weinheim/München, S. 71-86

Inglehart, Ronald (1989): Kultureller Umbruch. Wertewandel in der westlichen Welt, Frankfurt a.M. New York

Landon, H.C. Robbins (1988): 1791, Mozart's Last Year, London 
Mannheim, Karl (1976) (zuerst 1928): Das Problem der Generationen. In: Friedeburg, Ludwig v. (Hrsg.): Jugend in der modernen Gesellschaft, Köln, S. 23-48

Pfeil, Elisabeth (1968): Die 23jährigen. Eine Generationsuntersuchung am Geburtsjahrgang 1941, Tübingen

Rolff, Hans-G. (1980): Soziologie der Schulreform - Theorien, Forschungsberichte, Praxisberatung, Weinheim

Schelsky, Helmut (1957): Die skeptische Generation. Eine Soziologie der deutschen Jugend, Duisseldorf/ Köln 